University of Nebraska - Lincoln

DigitalCommons@University of Nebraska - Lincoln

\title{
Threshold-Related Effects on the High Energy Plateau in Above- Threshold Detachment
}

\author{
Katarzyna Krajewska \\ University of Nebraska-Lincoln, kkraj@fuw.edu.pl \\ Ilya I. Fabrikant \\ University of Nebraska-Lincoln, ifabrikant@unl.edu \\ Anthony F. Starace \\ University of Nebraska-Lincoln, astarace1@unl.edu
}

Follow this and additional works at: https://digitalcommons.unl.edu/physicsstarace

Part of the Physics Commons

Krajewska, Katarzyna; Fabrikant, Ilya I.; and Starace, Anthony F., "Threshold-Related Effects on the High Energy Plateau in Above-Threshold Detachment" (2007). Anthony F. Starace Publications. 100.

https://digitalcommons.unl.edu/physicsstarace/100

This Article is brought to you for free and open access by the Research Papers in Physics and Astronomy at DigitalCommons@University of Nebraska - Lincoln. It has been accepted for inclusion in Anthony F. Starace Publications by an authorized administrator of DigitalCommons@University of Nebraska - Lincoln. 


\title{
Threshold-Related Effects on the High Energy Plateau in Above-Threshold Detachment
}

\author{
K. Krajewska ${ }^{a, b}, *$ I. I. Fabrikant ${ }^{a, * *}$, and A. F. Starace ${ }^{a, * * *}$ \\ ${ }^{a}$ Department of Physics and Astronomy, The University of Nebraska, NE 68588-0111 Lincoln, United States \\ ${ }^{b}$ Institute of Theoretical Physics, Warsaw University, Hż 69, 00-681 Warszawa, Poland \\ *e-mail:kasia@unlserve.unl.edu \\ **e-mail: iif@unlserve.unl.edu \\ ***e-mail: astarace1@unl.edu \\ Received November 14, 2006
}

\begin{abstract}
We present results of a nonperturbative Floquet study of above-threshold detachment (ATD) of the $\mathrm{H}^{-}$and $\mathrm{F}^{-}$negative ions. Pronounced enhancements of ATD rates in the high-energy plateau region are found as the laser field intensity passes across ponderomotively shifted multiphoton thresholds. Depending on the symmetry of the initial state ( $s$ or $p$ symmetry), the enhancement is found to be most pronounced for even- or odd-channel closures, which is consistent with threshold laws applicable at the closing of particular multiphoton channels. Variations of ATD photoelectron angular distributions as functions of laser intensity near multiphoton thresholds are also investigated.
\end{abstract}

PACS numbers: 32.80.Rm, 32.80.Gc

DOI: $10.1134 / \mathrm{S} 1054660 \mathrm{X} 07040123$

\section{INTRODUCTION}

Photoelectron spectra obtained through high-intensity laser-matter interaction have received much attention in recent decades. Above-threshold ionization (ATI) or the related above-threshold detachment (ATD), which are considered to be among the most fundamental processes in strong field physics, proceed with absorption of many more additional photons than necessary to produce continuum electrons from atoms or ions (see [1]; for later reviews on ATI, see, e.g., [25]). This results in a sequence of peaks in the ATI and ATD photoelectron spectra that are separated by the photon energy and have nearly equal intensity over a large energy interval (the so-called "plateau" region). Although the key features of the ATI and ATD spectra can be successfully described by the "rescattering" (or "three step") semiclassical model [6, 7], more structured features of the spectrum, such as the resonant-like enhancements of the high energy part of the ATI spectrum reported in recent experiments for noble gases [813], require a quantum treatment.

The above-mentioned experiments [8-13] have shown that a subset of ATI high-energy plateau peaks can be raised significantly (by up to an order of magnitude) when the driving laser field intensity changes by only a few percent. Remarkably, up till now, there has been no consensus regarding the precise origin of such highly intensity-selective enhancements in the yield of plateau electrons [10-12, 14-24]. Basically, two distinct physical scenarios responsible for the occurrence of such enhancements have been proposed. One interpretation relates the enhancements to multiphoton resonances involving the ponderomotively upshifted excited states $[10,11,14,15,19,20,23]$, while an alternative explanation relates the resonant-like enhancements to laser-induced multiphoton channel closings (CCs) $[12,16-18,21,22$, 24], i.e., to a thresholdrelated effect. Since the ponderomotive potential raises the continuum threshold, the minimum number of photons required to ionize an electron from an atom (or detach an electron from a negative ion), $n$, increases when the laser intensity is increased, or, in other words, the $n$-photon channel for ionization or detachment can become closed as the laser field intensity increases. It is the purpose of this work to show that near the intensity at which this happens, a portion of the ATD rescattering plateau increases significantly and that the increase depends strongly on the electron's initial state symmetry ( $s$ or $p$ symmetry). In this view, the resonant-like enhancements of ATD (or ATI) high energy plateau electrons give evidence for the applicability of the Wigner threshold law [25] at the closing of multiphoton channels. Some aspects of threshold-related effects on the ATD electron angular distributions (ADs) are also discussed.

Throughout this paper, atomic units (a.u.) are used unless otherwise noted.

\section{THEORY}

In describing strong field detachment of negative ions, we assume the laser field to be a linearly polarized plane wave with its polarization vector $\hat{\boldsymbol{\varepsilon}}$ pointing in the $z$-direction, $\hat{\boldsymbol{\varepsilon}}=\hat{\mathbf{z}}$, and with its electric field and vector potential given by $\mathscr{F}_{(}(t)=\mathscr{F}_{0} \hat{\boldsymbol{\varepsilon}} \cos \omega t$ and $\mathscr{A}(t)=$ 
$\mathscr{A}_{0} \hat{\boldsymbol{\varepsilon}} \sin \omega t$, respectively, where $\omega$ is the frequency, $\mathscr{F}_{0}$ is the amplitude of the laser field, and $\mathscr{A}_{0}=-c \mathscr{F}_{0} / \omega$. Thus, the Hamiltonian $H$ of our system in the dipole velocity gauge has the form

$$
H(t)=H_{0}+\frac{1}{c} \mathscr{A}(t) \cdot \mathbf{p},
$$

where $H_{0}$ is the field-free Hamiltonian of the model ion that we treat within the single active electron (SAE) approximation [26]. In this approximation,

$$
H_{0}=\frac{\mathbf{p}^{2}}{2}+W,
$$

where $\mathbf{p}$ is the canonical momentum of the active (valence) electron and $W$ is an effective potential describing the electron-atom interaction. Due to the time periodicity of the Hamiltonian $H(t)$, one can use the Floquet theorem (for a review, see [28]) to represent the quasi-stationary state of the system by the so-called Floquet ansatz,

$$
|\Phi(t)\rangle=e^{-i E t} \sum_{n} e^{-i n \omega t}\left|\phi_{n}^{(E)}\right\rangle,
$$

where the index $n$ describes the net number of photons absorbed by the ion. The harmonic components $\left|\phi_{n}^{(E)}\right\rangle$ that belong to the quasi-energy $E$ are the solutions of the infinite system of coupled equations,

$$
\left(E+n \omega-U_{p}-H_{0}\right)\left|\phi_{n}^{(E)}\right\rangle=V_{+}\left|\phi_{n-1}^{(E)}\right\rangle+V_{-}\left|\phi_{n+1}^{(E)}\right\rangle,
$$

that originate from the time-dependent Schrödinger equation upon substitution of Eq. (3). Here, $U_{p}=$ $\left(\mathscr{F}_{0} / 2 \omega\right)^{2}$ is the ponderomotive energy of the electron quiver motion in the laser field, and $V_{+}$and $V_{-}$are the Fourier components of the dipole operator

$$
V_{+} e^{-i \omega t}+V_{-} e^{i \omega t}=\frac{1}{c} \mathscr{A}(t) \cdot \mathbf{p} .
$$

Equation (4) must be solved with appropriate boundary conditions imposed on the harmonic components at large distances from the atomic center,

$$
\left|\phi_{n}^{(E)}\right\rangle \sim \frac{e^{i k_{n} r}}{r}, \quad r \longrightarrow \infty
$$

where $k_{n}=\sqrt{2\left(E+n \omega-U_{p}\right)}$ is the wave number. The above boundary conditions cannot be satisfied unless the quasi-energy $E$ is complex. It can be represented as

$$
E=E_{0}+\Delta-\frac{i \Gamma}{2},
$$

where $E_{0}$ is the initial energy of the active electron, $\Delta$ describes the ac Stark shift of the initial state energy, and the imaginary part is related to the total decay rate, $\Gamma$, of the system (for more details, see, e.g., [27-31]).
Expressing the threshold energy in the presence of the laser field in units of the photon energy, $X \equiv(-\operatorname{Re} E+$ $\left.U_{p}\right) / \omega$, the minimum number of laser photons that are necessary to detach the electron is $n_{0}=1+[X]$ (unless $X=[X]$, in which case $n_{0}=X$ ). When the number of absorbed photons is such that $n \geq n_{0}$, or, in other words, $\operatorname{Re} E+n \omega-U_{p}>0$, the $n$-photon channel is open and the wave function for this harmonic component satisfies outgoing wave boundary conditions (cf. Eq. (6)); otherwise, the $n$-photon channel is closed.

The infinite system of coupled Eqs. (4) with boundary conditions given by Eq. (6) is solved by expanding the harmonic components on a discrete basis of complex radial Sturmian functions and spherical harmonics after truncation at some point. The numerical results are obtained by solving the resulting eigenvalue problem for quasi-energies and the coefficients of the Sturmian expansion (for details of numerical methods used in this context, see [32]). Once the eigenvalue problem is solved, the angular distributions of photoelectrons can be calculated. Since the theoretical formulation is provided in great detail elsewhere [27-31], we present here only a brief summary. For a linearly polarized laser field, the angular distribution $\frac{d \Gamma^{(n)}}{d \Omega}$ of photoelectrons that have been detached with absorption of $n \geq n_{0}$ photons from a state with quasi-energy $E$ and emitted into the solid angle $d \Omega$ with drift momentum $\mathbf{k}_{f}$ [where $k_{f}=$ $\left.\sqrt{2\left(\operatorname{Re} E+n \omega-U_{p}\right)}\right]$, is defined as

$$
\frac{d \Gamma^{(n)}}{d \Omega}=2 \pi k_{f}\left|M_{n}\left(\mathbf{k}_{f}\right)\right|^{2},
$$

where $M_{n}\left(\mathbf{k}_{f}\right)$ is the $n$-photon detachment amplitude,

$$
M_{n}\left(\mathbf{k}_{f}\right)=\sum_{N} e^{i(N-n) \beta} J_{N-n}(-\alpha)\left\langle\mathbf{k}_{f}|W| \phi_{E}^{(N)}\right\rangle,
$$

and where the real coefficients $\alpha$ and $\beta$ obey the relation

$$
\alpha \cos (\omega t-\beta)=\int^{t} d \tau \mathbf{k}_{f} \cdot \mathscr{A}(\tau)
$$

Integrating the angular distribution in Eq. (8) over all possible directions of the ejected electron, one obtains the partial rate $\Gamma^{(n)}$ for detachment into the $n$-photon channel

$$
\Gamma^{(n)}=\int \frac{d \Gamma^{(n)}}{d \Omega} d \hat{\mathbf{k}}_{f} .
$$

The partial rates sum up to the total detachment rate $\Gamma$. Since $\Gamma=-2 \operatorname{Im} E$ [cf. Eq. (7)], we arrive at the relation

$$
\sum_{n \geq n_{0}} \Gamma^{(n)}=-2 \operatorname{Im} E
$$




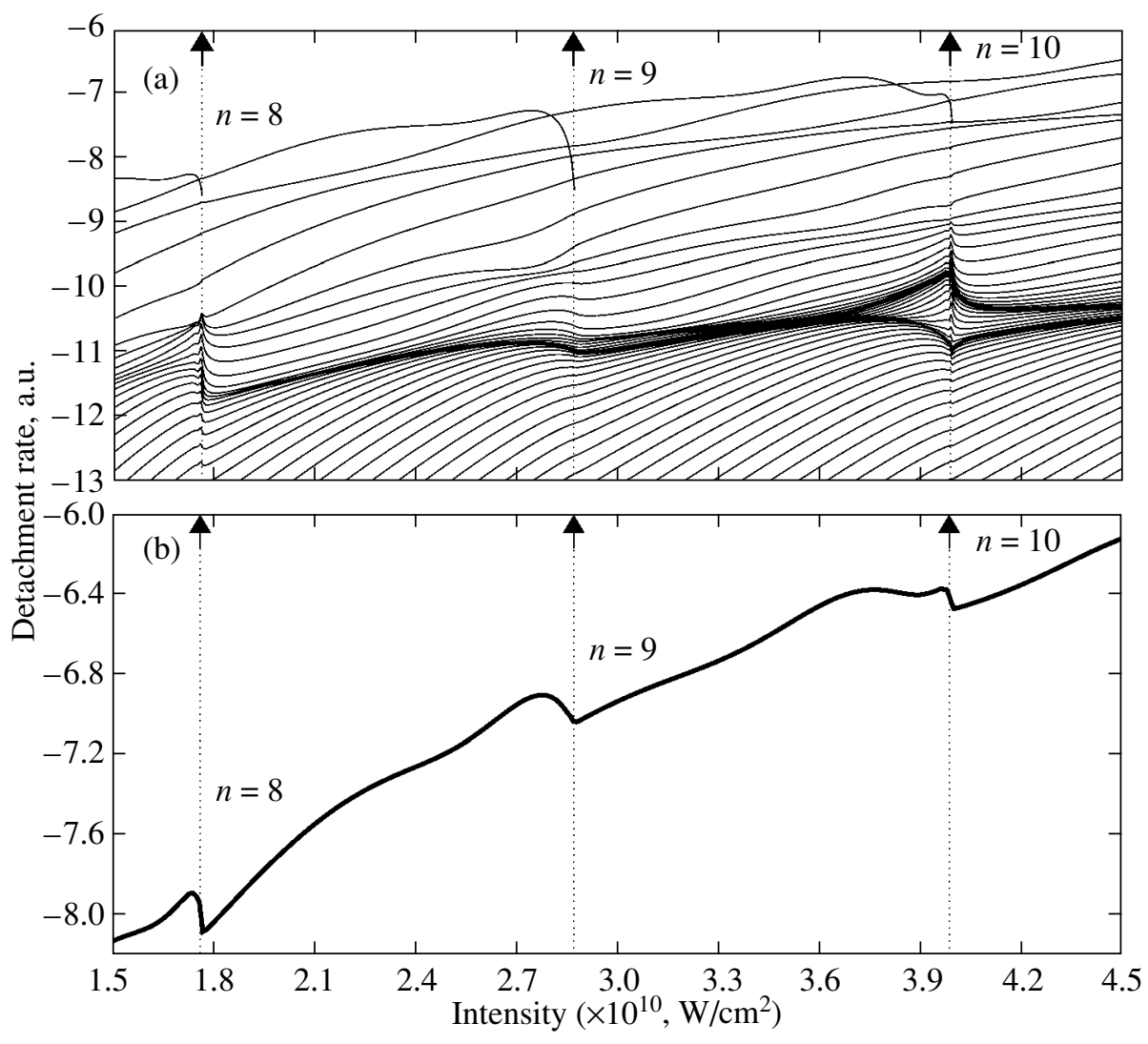

Fig. 1. (a) Partial rates $\Gamma^{(n)}$ as functions of the laser field intensity for $\mathrm{H}^{-}$detachment by a laser field of frequency 0.0043 a.u., in the intensity region where the eight-, nine-, and ten-photon detachment channels (marked by arrows) become closed due to the ponderomotive shift of the continuum limit (at laser intensities close to $1.76,2.87$, and $3.99\left(\times 10^{10} \mathrm{~W} / \mathrm{cm}^{2}\right)$, respectively). (b) Intensity dependence of the total detachment rate, $\Gamma$, for the same case as in panel (a). Different kinds of cusps [24, 33] are observed near intensities responsible for multiphoton channel closings (which are marked by arrows, as in panel (a)).

which serves as an independent test of the accuracy of our approach.

\section{ION MODELS}

In this paper, we investigate and contrast two processes of photodetachment by a linearly polarized laser field: (a) detachment of the hydrogen negative ion, $\mathrm{H}^{-}$, by a laser field of frequency $\omega=0.0043$ a.u. $(\lambda=$ $10.6 \mu \mathrm{m})$; and (b) detachment of the fluorine negative ion, $\mathrm{F}^{-}$, by a laser field of frequency $\omega=0.0253$ a.u. $(\lambda=1.8 \mu \mathrm{m})$. Within the SAE approximation, we describe each ion by a short range Yukawa potential

$$
W(r)=-W_{0} \frac{e^{-\lambda r}}{r}
$$

where the potential parameters employed are: $W_{0}=$ 1.1 a.u. for $\mathrm{H}^{-}, W_{0}=5.0745$ a.u. for $\mathrm{F}^{-}$, and $\lambda=1.0$ a.u. in both cases. (For more details regarding model potentials, see $[24,27]$.) With the chosen parameters for $\mathrm{H}^{-}$, the Yukawa potential in Eq. (13) supports only one bound state, which has an orbital angular momentum $l=0$ (i.e., an $s$-state) and energy $E_{0}=-0.0275654$ a.u. [27]. The model potential for describing $\mathrm{F}^{-}$, on the other hand, supports three bound states, with the valence state having $p$-symmetry (i.e., an orbital angular momentum $l=1)$ and a binding energy $E_{0}=-0.12498$ a.u. [24]. Note also that for weak laser intensities at least seven $10.6 \mu \mathrm{m}$ - and at least five $1.8 \mu \mathrm{m}$-photons have to be absorbed for $\mathrm{H}^{-}$and $\mathrm{F}^{-}$detachment, respectively. Those thresholds for photodetachment increase, however, with increasing laser intensities.

\section{THRESHOLD EFFECTS ON ENERGY AND ANGULAR DISTRIBUTIONS}

We start our analysis by presenting the intensity dependence of the ATD spectra for the $\mathrm{H}^{-}$and $\mathrm{F}^{-}$negative ions irradiated by linearly polarized laser fields with frequencies $\omega=0.0043$ and 0.0253 a.u., respectively. In Figs. 1a and 2a, we show the respective partial rates $\Gamma^{(n)}$, in a.u., as functions of the laser field intensity $I=c \mathscr{F}_{0}^{2} /(8 \pi)$ (given in units of W/cm²). In Fig. 1a, we show the $n$-photon detachment rates for the $\mathrm{H}^{-}$ion, $\Gamma^{(n)}$, 


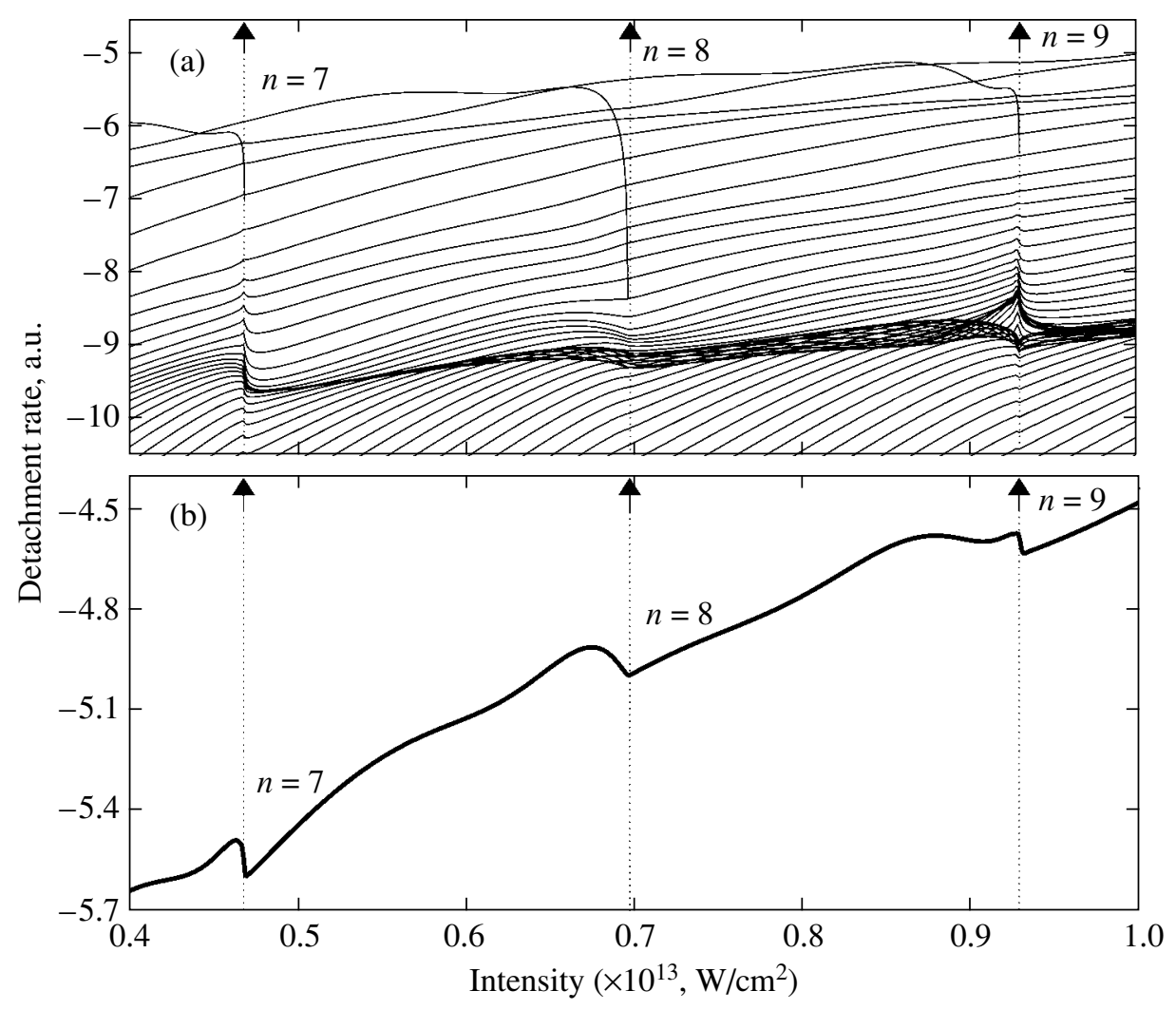

Fig. 2. The same as Fig. 1, but for the $\mathrm{F}^{-}$ion irradiated by a laser field of frequency $\omega=0.0253$ a.u., in the region of intensities over which the $n=7,8$, and 9 channels become closed (at intensities of the laser field close to $4.69,6.97$, and $9.30\left(\times 10^{12} \mathrm{~W} / \mathrm{cm}^{2}\right)$, respectively). The results for the partial rates (in panel (a)) and for the total rate (in panel (b)) have been averaged over the projections of the initial angular momentum, $l=1$.

for $8 \leq n \leq 60$ and for a range of laser field intensities such that the $n=8,9$, and 10 multiphoton detachment channels become closed. These closures occur at intensities close to $I=1.76,2.87$, and $3.99\left(\times 10^{10} \mathrm{~W} / \mathrm{cm}^{2}\right)$, respectively. One can observe a pronounced enhancement (up to an order of magnitude for laser-field intensities close to $3.99 \times 10^{10} \mathrm{~W} / \mathrm{cm}^{2}$ ) of groups of $n$-photon detachment rates near the even channel closures. As follows from Fig. 3, where we present the ATD spectra for the same case of $\mathrm{H}^{-}$detachment by a laser field with frequency 0.0043 a.u. but for fixed laser intensities chosen to span the ponderomotively shifted 8-, 9-, and 10photon detachment thresholds, the enhancement appears to be most pronounced in the lower- and midenergy part of the rescattering plateau, where the influence of the atomic potential is strongest.

In Fig. 2a, we display a similar intensity dependence of the partial rates $\Gamma^{(n)}$, for detachment of the $\mathrm{F}^{-}$ion (averaged over the projections of the angular momentum $l=1$ ) for channels $7 \leq n \leq 61$ in the region of intensities where, owing to the increasing ponderomotive shift with increasing intensity, the $n=7,8$, and 9 channels become closed. These closures occur at laser field intensities close to $I=4.69,6.97$, and 9.30 $\left(\times 10^{12} \mathrm{~W} / \mathrm{cm}^{2}\right)$, respectively. Comparing in Figs. 1a and
$2 \mathrm{a}$, one can notice that the enhancements for $\mathrm{H}^{-}$(having an $s$ valence electron with orbital angular momentum $l=0$ ) are pronounced for open channels at the even channel closures, while for $\mathrm{F}^{-}$(having a $p$ valence electron with orbital angular momentum $l=1$ ), the pronounced enhancements manifest themselves when the odd channels are closed. Such sensitivity of the ATD partial rates to both a small variation of the laser intensity and to the initial state symmetry of the valence electron agrees very well with the Wigner threshold law [25]. For short-range potentials, such as the one we work with [see Eq. (13)], the partial rates $\Gamma^{(n)}$ near the $m$-photon detachment threshold behave (unless $n=m$ ) like [25]

$$
\Gamma^{(n)} \simeq \text { const }+\mathscr{C}\left|E+m \omega-U_{p}\right|^{l_{\min }+1 / 2},
$$

with different values of $\mathscr{C}$ below and above threshold, and where $l_{\min }$ is the minimal angular momentum carried by the outer electron after its detachment with absorption of $m$-laser photons. According to the selection rules for a linearly polarized laser field, $l_{\min }$ can be 0 when the $s$ and $p$ electrons are detached with absorption of an even or odd number of photons, respectively. 
Detachment rate, a.u.

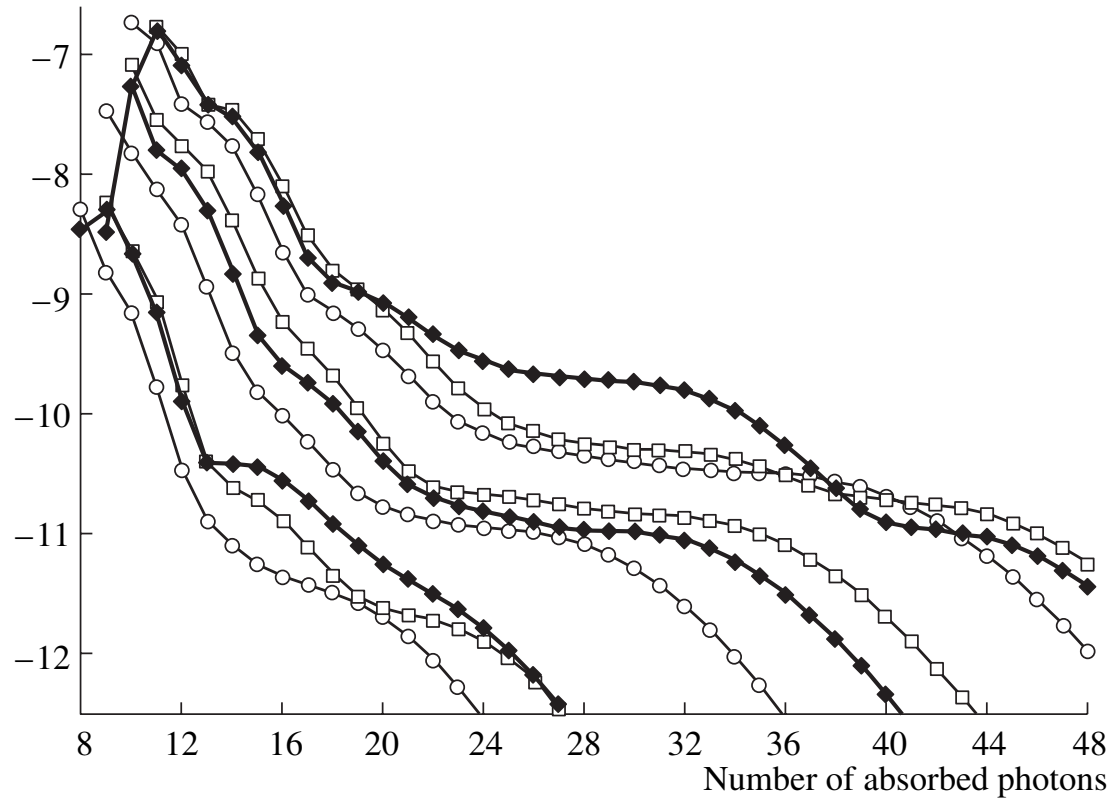

Fig. 3. ATD photoelectron spectra for $\mathrm{H}^{-}$detachment by a laser field at frequency 0.0043 a.u. and nine laser field intensities (in units of $10^{10} \mathrm{~W} / \mathrm{cm}^{2}$ ), with each set of three chosen to span each of the ponderomotively shifted eight-, nine-, and ten-photon detachment thresholds. Circles correspond to the three intensities below each of those thresholds (from bottom to top): 1.50, 2.47, and 3.69, while squares correspond to the three intensities slightly beyond the closure of each of those thresholds (from bottom to top): 1.80 , 3.14, and 4.09, respectively. Black diamonds indicate the three laser intensities (from bottom to top, 1.76, 2.87, and 3.99) that close the eight-, nine-, and ten-photon channels, respectively-i.e., they correspond to the intensities indicated by dotted lines and arrows in Fig. 1.

Thus, the dominant term in $\Gamma^{(n)}$ simply has the form $\Gamma^{(n)} \sim \sqrt{\left|E+m \omega-U_{p}\right|}$, leading to singularities of the first derivative of $\Gamma^{(n)}$ with respect to intensity (or, equivalently, to $U_{p}$ ) at the closing of the $m$-photon channel. This can be seen in the partial rates presented in Figs. 1a and 2a in the form of different kinds of cusps that have been predicted within the theory of nuclear reactions [33], and have recently been reinvestigated for multiphoton detachment [24]. Consequently, the total rates $\Gamma$ for $\mathrm{H}^{-}$and $\mathrm{F}^{-}$detachment as functions of the laser intensity displayed in Figs. $1 \mathrm{~b}$ and $2 \mathrm{~b}$ of the same figures also give evidence for CCs.

Comparing Figs. 1a and 2a, one observes that for fixed intensity of the laser field, the ATD partial rates for $s$ - and $p$-electron detachment processes show qualitatively different behavior in the plateau region. While both have nearly equal rates for different values of $n$ on the plateau, for the $s$-electron initial state, the rates form a monotonically decreasing, dense structure as a function of increasing $n$, whereas in the case of the $p$ initial state, the rates cross each other. These rate crossings produce a much more complex pattern in the highenergy ATD plateau for the $p$ - than for the $s$-symmetry initial electron state, which may be seen by comparing Figs. 1a and 2a for fixed values of intensity. When the ATD rates are plotted against increasing $n$ for fixed laser intensity, as in Figs. 3 and 4 for $\mathrm{H}^{-}$and $\mathrm{F}^{-}$, respec- tively, one sees that the curves for $\mathrm{H}^{-}$are monotonically decreasing, while those for $\mathrm{F}^{-}$have various maxima and minima, even for intensities not at the channel closings. Because of this nonmonotonic variation of the ATD rates for the case of an initial $p$-state symmetry with increasing number of absorbed laser photons, $n$, at fixed laser intensity, it is more difficult to distinguish plateau enhancements by examining the ATD spectra for only a few laser intensities that span the ponderomotively shifted thresholds (cf. Fig. 4). In general, in order to recognize the strong field enhancement unambiguously, one should obtain the ATD spectra over a wide range of finely spaced values of laser intensities, as in Figs. 1a and 2a.

As CCs affect both the total and partial detachment rates, one expects to observe signatures of CCs in photoelectron angular distributions [24, 34-41]. The sensitivity of two- and three-photon angular distributions to variations of the laser intensity near detachment thresholds have been studied both experimentally [34, 37] and theoretically $[35,36,41]$ for $\mathrm{H}^{-}$. The angular distribution of ATD electrons for $\mathrm{F}^{-}$[38-40], as well as for other halogen negative ions [40], have been investigated for fixed laser intensity. Only recently [24] have the variations of 8- and 9-photon $\mathrm{F}^{-}$detachment $\mathrm{ADs}$ been investigated as a function of laser intensity in the vicinity of CCs. With reference to this work [24], we 
Detachment rate, a.u.

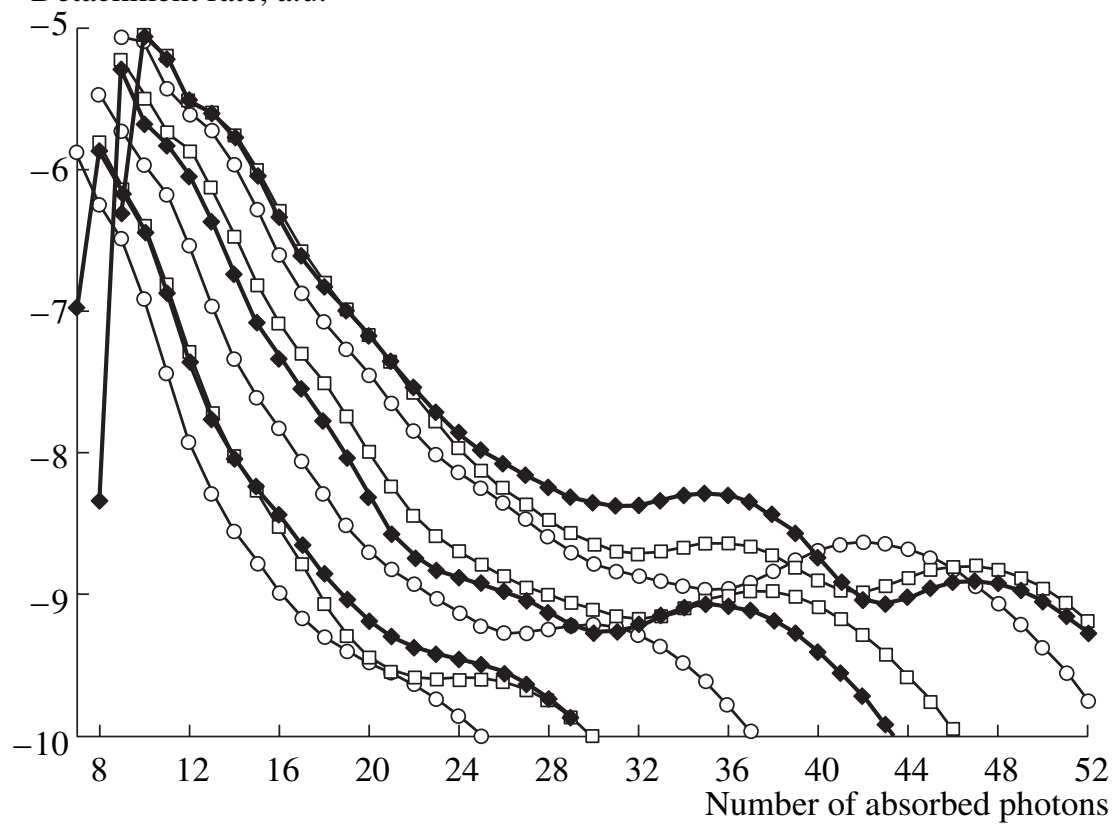

Fig. 4. The same as Fig. 3, but for $\mathrm{F}^{-}$detachment by a linearly polarized laser field at frequency 0.0253 a.u. As in Fig. 2 , the results have been averaged over the initial angular momentum projections. The three sets of three curves (from bottom to top) correspond to laser intensities (in units of $10^{12} \mathrm{~W} / \mathrm{cm}^{2}$ ) that span the ponderomotively shifted seven-, eight-, and nine-photon detachment thresholds, respectively. Circles indicate intensities 4.0, 5.92, and 8.7 (from bottom to top), squares indicate intensities 4.8, 7.5, and 9.4 (from bottom to top), while black diamonds correspond to intensities 4.69, 6.97, and 9.30 (from bottom to top) that are indicated by arrows in Fig. 2.

analyze here the intensity dependence of ADs near the 8- and 9-photon $\mathrm{CCs}$ for $\mathrm{H}^{-}$detachment.

Figure 5 presents the intensity dependence of the 8- and 9-photon ADs (Fig. 5a, respectively) for $\mathrm{H}^{-}$ detachment by a linearly polarized laser field of frequency $\omega=0.0043$ a.u. for cases when the laser intensity approaches the values corresponding to the closing of these particular thresholds. In Figs. 5a and 5b, we see that while for a smaller laser intensity the electron is preferably emitted along the polarization vector of the laser field $\hat{\boldsymbol{\varepsilon}}$ (i.e., along $0^{\circ}$ or $180^{\circ}$ ), when the laser intensity changes by only a few percent towards closure of the eight-photon detachment channel, the shape of the $\mathrm{AD}$ changes dramatically, with the largest maximum pointing perpendicular to the laser field polarization direction. In particular, for the intensity $1.7 \times$ $10^{10} \mathrm{~W} / \mathrm{cm}^{2}$, the AD has a bell shape, as was also found in $[34,36,41]$ for two-photon detachment of $\mathrm{H}^{-}$near the two-photon threshold. As one approaches the eightphoton threshold, the AD becomes isotropic, as shown

Anisotropy parameters $\beta_{2 l}$ for eight- and nine-photon detachment of $\mathrm{H}^{-}$by a laser field with frequency 0.0043 a.u. for the same four laser intensities as in Figs. 5a and 5b, respectively. Bracketed numbers indicate powers of 10

\begin{tabular}{l|c|c|c|c|c|c|c|c}
\hline & \multicolumn{7}{|c}{ Laser field intensity $\left(\times 10^{10} \mathrm{~W} / \mathrm{cm}^{2}\right)$} \\
\hline & \multicolumn{7}{|c|}{$n=8$} & \multicolumn{4}{c}{$n=9$} \\
\hline & 1.5 & 1.6 & 1.7 & 1.76 & 2.57 & 2.77 & \multicolumn{1}{c}{2.85} & 2.87 \\
\hline$\beta_{2}$ & $6.40[-1]$ & $-8.11[-2]$ & -1.48 & $2.89[-2]$ & $-6.77[-1]$ & 1.07 & 1.80 & 1.95 \\
$\beta_{4}$ & 1.86 & 2.07 & $5.28[-1]$ & $1.36[-4]$ & -1.21 & -1.04 & $-2.55[-1]$ & $-6.92[-2]$ \\
$\beta_{6}$ & -1.12 & $-5.70[-2]$ & $-4.63[-2]$ & $-7.95[-8]$ & 2.10 & $2.21[-1]$ & $1.13[-2]$ & $8.11[-4]$ \\
$\beta_{8}$ & $2.14[-1]$ & $6.03[-2]$ & $1.75[-3]$ & $9.85[-8]$ & $-7.28[-1]$ & $-1.94[-2]$ & $-2.30[-4]$ & $-4.45[-6]$ \\
$\beta_{10}$ & $-1.94[-2]$ & $-3.21[-3]$ & $-3.51[-5]$ & $-1.21[-7]$ & $1.13[-1]$ & $8.98[-4]$ & $2.46[-6]$ & $1.84[-8]$ \\
$\beta_{12}$ & $9.58[-4]$ & $9.74[-5]$ & $4.58[-7]$ & $-2.41[-8]$ & $-9.63[-3]$ & $-2.49[-5]$ & $-9.92[-8]$ & $-4.64[-8]$ \\
\hline
\end{tabular}

LASER PHYSICS Vol. $17 \quad$ No. 42007 


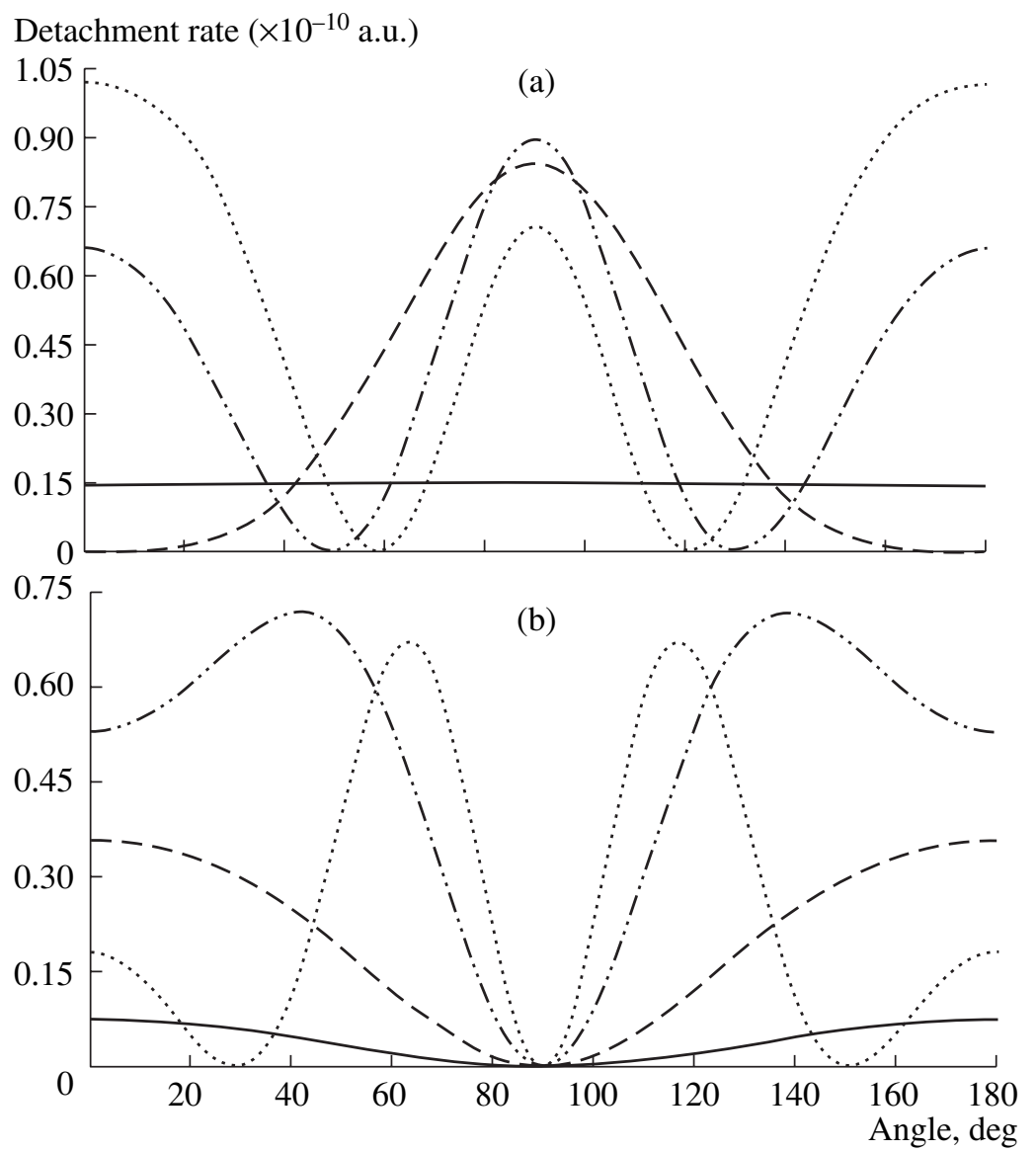

Fig. 5. The ADs for eight- and nine-photon detachment of $\mathrm{H}^{-}$(panels (a) and (b), respectively) by a linearly polarized laser field of frequency $\omega=0.0043$ a.u. for different laser intensities (given here in units of $10^{10} \mathrm{~W} / \mathrm{cm}^{2}$ ). (a) Each curve corresponds to a laser intensity near the eight-photon channel closure: $I=1.5$ (dotted line), 1.6 (dash-dot-dotted line), 1.7 (dashed line), and 1.76 (solid line). (b) Same as in panel (a), but for laser field intensities near the ponderomotively shifted nine-photon detachment threshold: $I=$ 2.57 (dotted line), 2.77 (dash-dot-dotted line), 2.85 (dashed line), and 2.87 (solid line). Note the sudden drop of the eight- and ninephoton detachment rates (by almost an order of magnitude) as one reaches the channel closing laser intensity.

in Fig. 5a, for the intensity $1.76 \times 10^{10} \mathrm{~W} / \mathrm{cm}^{2}$. In contrast, for nine-photon detachment and laser intensities close to this channel closure, there are no electrons ejected in the direction perpendicular to the laser polarization vector, and the ADs become peaked along the polarization vector $\hat{\boldsymbol{\varepsilon}}$ as the laser intensity increases. Similarly, dramatic changes in the shapes of the ADs for other even multiphoton detachment channels of $\mathrm{H}^{-}$ as a function of laser field intensity in the vicinity of the channel closings have been found in our calculations, while the more typical or expected ADs found for the nine-photon channel have also been found for other odd multiphoton channels. As we have shown in [24], the behaviors of the ADs for odd and even photon channels for $\mathrm{F}^{-}$are reversed from those for $\mathrm{H}^{-}$.

In order to gain more insight into the structure of the ADs displayed in Fig. 5, we conclude by investigating a case of partial wave mixing noted by Telnov and Chu in [36]. The treatment is based on an expansion of the
AD in terms of the Legendre polynomials $P_{2 l}(\cos \theta)$, which depend on the angle $\theta$ between the electron momentum, $\mathbf{k}_{f}$, and the laser polarization, $\hat{\boldsymbol{\varepsilon}}$ :

$$
\frac{d \Gamma^{(n)}}{d \Omega}=\frac{\Gamma^{(n)}}{4 \pi}\left(1+\sum_{l=1}^{\infty} \beta_{2 l} P_{2 l}(\cos \theta)\right) .
$$

Here, $\beta_{2 l}$ are the anisotropy parameters, which are given in the table for each AD plotted in Fig. 5. One can observe dramatic changes in the contributions of different partial waves to the eight- and nine-photon detachment ADs with varying laser intensity in the vicinity of the channel closings. With increasing intensity, the contributions of $s$ and $p$ waves become dominant for the eight- and nine-photon detachment channels, respectively. However, even only slightly below those thresholds, the ADs result from a superposition of different partial waves. 


\section{CONCLUSIONS}

In summary, we have demonstrated pronounced enhancements of the high-energy region of the ATD plateau for both $\mathrm{H}^{-}$and $\mathrm{F}^{-}$ions for laser intensities in the vicinity of even or odd photon detachment channel closings, respectively. We have shown that the sensitivity of ATD rates to the initial state symmetry of the active electron is consistent with the Wigner threshold law applicable at multiphoton CCs. Also, we have presented variations of the photoelectron angular distributions in the vicinity of the channel closings.

\section{ACKNOWLEDGMENTS}

One of us (K.K.) gratefully acknowledges support from the Swiss National Science Foundation under SCOPES grant no. IB7120-114584 LPHYS'06. This work was supported in part by the Polish Committee for Scientific Research under grant no. KBN 1 P03B 006 28 (K.K.), the U.S. National Science Foundation under grant nos. PHY-0354688 (I.I.F.) and PHY-0601196 (A.F.S.), and by a Jorgensen Postdoctoral Fellowship at the University of Nebraska-Lincoln (K.K.). A.F.S. gratefully acknowledges partial support from the Alexander von Humboldt Stiftung and the Max-PlanckInstitut für Quantenoptik (MPQ), as well as the hospitality of the MPQ, where part of this manuscript was prepared.

\section{REFERENCES}

1. P. Agostini, F. Fabre, G. Mainfray, et al., Phys. Rev. Lett. 42, 1127 (1979).

2. L. F. DiMauro and P. Agostini, Adv. At. Mol. Opt. Phys. 35, 79 (1995).

3. M. Protopapas, C. H. Keitel, and P. L. Knight, Rep. Prog. Phys. 60, 389 (1997).

4. N. B. Delone and V. P. Krainov, Phys. Usp. 41, 469 (1998).

5. W. Becker, F. Grasbon, R. Kopold, et al., Adv. At. Mol. Opt. Phys. 48, 35 (2002).

6. K. J. Schafer, B. Yang, L. F. DiMauro, and K. C. Kulander, Phys. Rev. Lett. 70, 1599 (1993).

7. P. B. Corkum, Phys. Rev. Lett. 71, 1994 (1993).

8. P. Hansch, M. A. Walker, and L. D. Van Woerkom, Phys. Rev. A 55, R2535 (1997).

9. M. P. Hertlein, P. H. Bucksbaum, and H. G. Muller, J. Phys. B 30, L197 (1997).

10. M. J. Nandor, M. A. Walker, L. D. Van Woerkom, and H. G. Muller, Phys. Rev. A 60, R1771 (1999).

11. E. Cormier, D. Garzella, P. Breger, et al., J. Phys. B 34, L9 (2000).

12. G. G. Paulus, F. Grasbon, H. Walther, et al., Phys. Rev. A 64, 021401(R) (2001).

13. F. Grasbon, G. G. Paulus, H. Walther, et al., Phys. Rev. Lett. 91, 173003 (2003).
14. H. G. Muller and F. C. Kooiman, Phys. Rev. Lett. 81, 1207 (1998).

15. H. G. Muller, Phys. Rev. A 60, 1341 (1999).

16. R. Kopold, W. Becker, M. Kleber, and G. G. Paulus, J. Phys. B 35, 217 (2002).

17. B. Borca, M. V. Frolov, N, L. Manakov, and A. F. Starace, Phys. Rev. Lett. 88, 193001 (2002).

18. S. V. Popruzhenko, Ph. A. Korneev, S. P. Goreslavski, and W. Becker, Phys. Rev. Lett. 89, 023001 (2002).

19. J. Wassaf, V. Véniard, R. Taïeb, and A. Maquet, Phys. Rev. Lett. 90, 013003 (2003).

20. J. Wassaf, V. Véniard, R. Taïeb, and A. Maquet, Phys. Rev. A 67, 053405 (2003).

21. K. Krajewska, I. I. Fabrikant, and A. F. Starace, Bull. Am. Phys. Soc. 51, 119 (2006).

22. N. L. Manakov and M. V. Frolov, JETP Lett. 83, 536 (2006).

23. R. M. Potvliege and S. Vučić, Phys. Rev. A 74, 023412 (2006).

24. K. Krajewska, I. I. Fabrikant, and A. F. Starace, Phys. Rev. A 74, 053407 (2006).

25. E. P. Wigner, Phys. Rev. 73, 1002 (1948).

26. K. C. Kulander, K. J. Schafer, and J. L. Krause, in Atoms in Intense Laser Fields, Ed. by M. Gavrila (Academic, Boston, 1992), p. 247.

27. R. Shakeshaft and X. Tang, Phys. Rev. A 36, 3193 (1987).

28. R. M. Potvliege and R. Shakeshaft, in Atoms in Intense Laser Fields, Ed. by M. Gavrila (Academic, Boston, 1992), p. 373.

29. R. M. Potvliege and R. Shakeshaft, Phys. Rev. A 38, 4597 (1988).

30. R. M. Potvliege and R. Shakeshaft, Phys. Rev. A 38, 6190 (1988).

31. R. M. Potvliege and R. Shakeshaft, Phys. Rev. A 40, 3061 (1989).

32. R. M. Potvliege, Comput. Phys. Commun. 114, 42 (1998).

33. A. I. Baz', Sov. Phys. JETP 6, 709 (1958).

34. R. Reichle, H. Helm, and I. Yu. Kiyan, Phys. Rev. Lett. 87, 243001 (2001).

35. B. Borca, M. V. Frolov, N. L. Manakov, and A. F. Starace, Phys. Rev. Lett. 87, 133001 (2001).

36. D. A. Telnov and S.-I. Chu, Phys. Rev. A 66, 063409 (2002).

37. R. Reichle, I. Yu. Kiyan, and H. Helm, J. Mod. Opt. 50, 461 (2003).

38. M. V. Frolov, N. L. Manakov, E. A. Pronin, and A. F. Starace, Phys. Rev. Lett. 91, 053003 (2003).

39. M. V. Frolov, N. L. Manakov, E. A. Pronin, and A. F. Starace, J. Phys. B 36, L419 (2003).

40. A. Gazibegović-Busuladžić, D. B. Milošević, and W. Becker, Phys. Rev. A 70, 053403 (2004).

41. L. Bai, J. Zhang, X. Zhang, and Z. Xu, Phys. Rev. A 74, 025402 (2006). 